\title{
Investigating student ownership of projects in an upper-division physics lab course
}

\author{
Jacob T. Stanley, ${ }^{1}$ Dimitri R. Dounas-Frazer, ${ }^{1}$ Laura Kiepura, ${ }^{1}$ and H. J. Lewandowski ${ }^{1,2}$ \\ ${ }^{1}$ Department of Physics, University of Colorado, Boulder, CO 80309, USA \\ ${ }^{2}$ JILA, National Institute of Standards and Technology and University of Colorado, Boulder, CO, 80309, USA
}

\begin{abstract}
The development of students' sense of ownership of their work is recognized by many lab instructors to be an important outcome of lab courses. However, the way ownership manifests, as well as how it is developed, has not been a focus of study within the physics education research community. As a first step toward understanding what ownership looks like in this context, we are studying students' ownership of their projects in two upper-division optics courses, in which ownership is an explicit learning goal. We utilized data from the Project Ownership Survey (POS), as well as student interviews that focus on their interests, challenges, and memorable moments. The results of the POS were conflicted - one portion of the survey indicated high ownership while those questions pertaining to student affect indicated otherwise. However, analysis of our interviews corroborated that students were experiencing several aspects of ownership, but the nature of their affective response was complex and dynamic.
\end{abstract}

\section{INTRODUCTION}

Promoting students' sense of ownership over projects is an important aspect of learning, both in instructional [1] and research settings [2. However, for many instructors and researchers, what it means to have a sense of ownership may be understood only in broad and surface-level terms [1]. In physics, one promising venue for engaging students in projects over which they feel ownership, is the instructional laboratory course, an environment which has not been well studied [3].

There have been a number of studies that focus on ownership in various science education environments [1, 4 9], including introductory physics courses for nonmajors 7 9]. However, we are unaware of work that focuses on student ownership of projects in upper-division physics lab courses. Drawing on the synthesis work of Wiley [1, students' ownership can be defined as consisting of three aspects: feeling responsible for, committed to, and personally connected to the project [1, 4]. Hanauer et al. 4, 5] have argued that this definition of ownership is related to five hallmarks of students who experience ownership: such students (i) are interested in the project, (ii) demonstrate agency in manging the project, (iii) express positive affect, such as excitement, about the project, (iv) demonstrate willingness to contend with challenges, and (v) work productively with others. However, how ownership of one's project manifests may depend largely on the context in which it's experienced. Here, we use the definition and hallmarks of ownership described by Wiley[1] and Hanauer et al. [4] as our framework to investigate how ownership manifests for students working on projects in the context of two upper-division physics lab courses. Ultimately, we find the framework does not fully capture their experiences.

In the present work, we draw on three data sources to gain insight into ownership: pre-project interviews, post-project interviews, and the Project Ownership Survey (POS) [5]. The POS, which is a quantitative measure of student ownership, was developed and validated in the context of the work that produced the framework we've adopted, and was intended to be used in introductory Course-based Undergraduate Research Experiences [4, 5]. Because the POS has not previously been administered in an upper-division physics context, we designed our interview protocols to probe for additional evidence that the students were experiencing ownership. By comparing and contrasting the results of the interviews to the POS results, we will show that the students in our study demonstrated several of the hallmarks of ownership. However, the POS items that focus on students' affect, which have been a useful measure of ownership in other contexts [5], do not adequately capture the complex affect dynamics experienced by the students in our study. We outline how these affect dynamics are associated with the struggles and successes of their projects.

\section{CONTEXT AND PARTICIPANTS}

We studied two related upper-division physics laboratory courses: the "Lasers" and "Optics" courses. Both focused on topics in contemporary optics, were designed and taught by the same instructor, and had the same structure for activities and projects. Due to their similarities, we did not compare between courses, and instead aggregated their data. These courses were offered at Bethel University - a private, four-year, Mastersgranting, predominantly white institution with enrollment of 6,500 .

As articulated by the instructor, course learning goals included development of students' technical skills, appreciation of the importance of lasers and optics in science and industry, excitement about experimental physics, and ownership of their final projects. Each course lasted 14 weeks: during the first half, students engaged in guided experimental skill-building activities and during the second half, students worked on final projects in groups of 2-4 people. Students were presented with a portfolio of possible projects and ranked each project according to their interest. Groups were formed based on students' project preference, and each was assigned to their first or second choice.

In total, there were 12 distinct projects, all of which 
were informed by the instructor's own research. As an example, one project was the continued development of a frequency comb - a useful tool for measuring optical frequencies found in many modern atomic, molecular, and optical physics experiments. During the second half of the course, there were no lab guides to reference for guidance on their projects. Instead, students were provided relevant scientific journal articles. There was no scheduled lab time; groups coordinated their own time management and division of labor. Students spent 10-15 hours per week working on their projects. Projects culminated in oral presentations and reports written in the style of a journal article. Based on the course design, we anticipated students would demonstrate many ownership hallmarks during the project portion of the courses.

The courses enrolled a total of 34 unique students, all of whom participated in the study. Two students were in both classes, resulting in a total of 36 participants. Most of the students had some prior experience in a lab setting similar to the two courses: 22 had taken both a previous upper-division lab course and had research experience, 10 had only taken a previous upper-division lab course, and one student had only research experience.

\section{METHODS}

We administered the POS during the final week of each course, and all 36 participants completed it. The POS consists of 16 five-point Likert items, which are split into two parts: items 1-10 address students' interest, agency, responsibility, challenges; items 11-16 address students' positive affect. For each survey item, we computed the mean and standard error of the student responses. The wording of each item and the corresponding Likert scales can be seen in Table 【.

The interview protocols and analysis scheme were both designed to provide insight into the presence of three ownership hallmarks: interest, agency, and affect. This allowed us to contextualize/interpret the POS results.

Pre/post-project interviews were conducted during the first/final weeks of the projects, were done using video conference or in person, and lasted 10-25 minutes. In total, 33 students participated in both pre- and postinterviews; 2 participated only in the post-interview; and 1 participated in neither. Interviews were transcribed and all analysis was performed on the transcriptions. Pre-interviews focused on students' physics education prior to the lab course and their interest in and excitement for the projects. Post-interviews focused on the evolution of students' interest, project challenges and how they were addressed, memorable moments, and preference for projects or guided activities.

Informed by our research goals, we identified the portions of the interview protocols that were anticipated to elicit responses germane to interest, agency, and affect. These questions (enumerated below) were then independently coded by authors two and three, who then discussed and reconciled any discrepancies in their codes.
1. pre: Can you give a little background about why this project is interesting to you?"

2. post: "Looking back on your experience in this class, has your interest in the project changed? If so, how?"

3. post: "What were some especially memorable moments during this lab experience?"

4. post: "What were some of the challenges, and how did they get resolved?"

5. post: "Did you prefer the exercises or the projects, and why?"

Students' interest and how it evolved were extracted from questions 1 and 2. The coding of students' reason for interest was emergent and was subsequently grouped into several categories. Students' change in interest was coded as having increased, stayed the same/fluctuated, or decreased, and their reasons were recorded.

Statements of students' agency were extracted from questions 3 and 4 . Our coding definition for agency consisted of instances when students were proactive in setting goals for their project, strategizing/planning in how to achieve a goal, or revising their plan or goal in response to challenges during the course of their project.

Students' affect was also extracted from questions 3 and 4. Along with specific descriptions of events, these questions elicited description of how the students felt during the experience. All coding of affect was emergent and was done by identifying words associated with an affective response: excitement, frustration, surprise, worry, happiness, etc. These were then grouped into two categories: "positive" or "negative" affect.

Finally, we coded question 5 for whether students preferred the project or activities and their reasoning. This helped synthesize findings about the three hallmarks.

\section{RESULTS AND INTERPRETATION}

In this section, we report the results of the POS and the interview coding. Calculated from the results shown in Table I] the weighted average (and standard error) of the class aggregate scores on items $1-10$ was $1.0 \pm 0.1$. This is at the high end of the range, indicating general agreement with these statements. On the other hand, the weighted average of items $11-16$ was $-0.1 \pm 0.1$. This is in the middle of the range, indicating a neutral response. Hanauer and Dolan found that all 16 items discriminated between "high ownership" and "low ownership" courses [5]. However, our scores on items 1-10 are consistent with Hanauer's high ownership courses, whereas items 11-16 are consistent with Hanauer and Dolan's low ownership courses. This discrepancy makes our POS results difficult to interpret. To understand this discrepancy, we turn to the coding results from the interview data.

According to Hanauer's framework, for students to develop a high sense of ownership, they should have initial interest in some aspect of their project that should develop over the course of the project; they must be given the opportunity to have agency over some aspect of their 


\begin{tabular}{llr}
\hline \hline No. & POS question & Mean $(\mathrm{SE})$ \\
\hline 1 & I had a personal reason for choosing the research project I worked on. & $0.6(0.2)$ \\
2 & My research project was interesting. & $0.5(0.1)$ \\
3 & My research will help solve a problem in the world. & $1.2(0.1)$ \\
4 & I faced challenges that I managed to overcome in completing my research project. & $1.3(0.1)$ \\
5 & My research project was exciting. & $1.1(0.1)$ \\
6 & I was responsible for the outcome of my research. & $1.2(0.2)$ \\
7 & The research question I worked on was important to me. & $0.6(0.2)$ \\
8 & The findings of my research project gave me a sense of personal achievement. & $1.2(0.1)$ \\
9 & In conducting my research project, I actively sought advice and support. & $1.4(0.1)$ \\
10 & My findings were important to the scientific community. & $-0.1(0.2)$ \\
\hline 11 & To what extent does the word surprised describe your experience in the laboratory course? & $0.2(0.2)$ \\
12 & To what extent does the word astonished describe your experience in the laboratory course? & $-0.3(0.2)$ \\
13 & To what extent does the word amazed describe your experience in the laboratory course? & $-0.4(0.2)$ \\
14 & To what extent does the word happy describe your experience in the laboratory course? & $0.1(0.2)$ \\
15 & To what extent does the word joyful describe your experience in the laboratory course? & $0.0(0.2)$ \\
16 & To what extent does the word delighted describe your experience in the laboratory course? &
\end{tabular}

TABLE I. POS items and average scores (standard error). Likert scales for items 1-10 ranged from Strongly agree (+2) to Strongly disagree $(-2)$. The average score for items $1-10$ was $1.0 \pm 0.1$. Likert scales for items $11-16$ ranged from Very strongly $(+2)$ to Very slightly $(-2)$. The average score for items $11-16$ was $-0.1 \pm 0.1$.

projects; and they should generally experience positive affect about their project as a whole [4. We now present evidence for these three hallmarks of ownership (interest, agency, and affect) in the interviews.

First, we consider students' interest. We determined all 33 students expressed interest in their project. 31 of those stated a specific reason for their interest, and 28 stated more than one. The reasons for interest fell into the following categories: (i) interest in the process of experimental physics, such as doing "hands on" experimentation, designing/building apparatus, or using specific equipment; (ii) general interest in the nature of research, such as working on a project that is "new" or "novel" with potential applications/scientific value; (iii) interest due to prior connection, such as having previously worked on a related project; and (iv) interest that centered on personal relationships with peers/instructors.

By the end of the project, almost all students (32 of 35 ) indicated that their interest had increased (19) or stayed the same (13). Of those who's interest stayed the same, all but one expressed initial interest in the project. Students who had increased interest described that this was due to their improved understanding of the purpose/value of the projects or having achieved successful results. For example:

"My interest has increased because I can see the scope for what [the project] can be used for now. Before I had known about it and I was interested in it, but now I can see how this project could be used for like really real applications, so I would say [my interest has] increased. And, yeah, also ownership of it has made it increase as well."

Furthermore, students whose interest stayed the same described that this was because their initial interest was high to begin with. For example:
"I think I was pretty interested to come into it from the start. Before this I had some experience in nanotechnology already and so I think I kind of saw this as a little bit of an extension ... and so I think I came in with it and am leaving with a high amount of interest."

Only three stated that their interest had decreased by the end. This was due to frustration and the challenges the project presented. Thus, we conclude that the students, in general, had a high degree of interest in the course that tended to grow as the project progressed. This is consistent with the POS results.

Second, we found that 30 students described instances where they exhibited agency, half of whom described such instances in response to both questions. Predominantly, students described formulating strategies to achieve a set goal, or adjusting these strategies in light of unexpected challenges. For example:

"An especially memorable one was when we found out that the two split off infrared beams were not being collimated. I suggested that we just take off [the] filter to help with our alignment, and it went a bit faster after we did that."

Only four students did not describe an instance where they exhibited agency. Thus, students seemed willing/able to take agency over their projects, consistent with the POS results.

Third, we found that 28 students expressed positive affect (namely, excited or happy) to describe their experiences with the project. Conversely, 9 students expressed negative affect (namely, frustrated or worried) to describe their experience - however, all but one expressed positive affect as well. Only five students did not express any affect in their responses. Generally, the positive affect was 
attributed to students getting good results or achieving an incremental goal. Negative affect was attributed to difficulties in making progress or struggling to get equipment working. The range of affect can clearly be seen in the following example.

"I think there have been times where we've had big successes that were exciting. For example, there's a piece that we milled out of a block of aluminum. When that was completed, we felt really satisfied with it. We were excited about that. There were times that were really frustrating. For example, we had designed something ... and we tried [making] it like 10 times and none of them worked ... It was really frustrating to not make any progress there. Those were the two most memorable things."

Although we found most students felt quite positive, for some of them, the frustrations that can accompany challenging and involved projects was also central to the experience. Therefore, the presence of both positive and negative affect make the POS results difficult to interpret, since the POS affect items are only positive.

As further evidence of students' ownership, we found 28 students preferred the project portion of the courses to the guided exercises. Students articulated various reasons for this preference. Consider the following example.

I definitely preferred the projects because it was open-ended. We were given a problem and kind of a direction to shoot for, and then kind of let loose. You really could go in a lot of different directions with it ... It led to a very good fluctuation in emotion throughout the entire thing. You know, you'd be really frustrated that something's not working, but then you'd have really great success, and the joy that came with that was just so much greater than anything that I've had in normal lab before. While it was time consuming ... it felt more like something that I wanted to do instead of something that I had to do."

This student stated being "let loose" on an "open-ended" project, indicating their ability to have agency over strategic decisions. They also described "good fluctuation in emotion" consisting of frustration and joy, indicating the importance of affect in their experience. Also, they stated the project was something they "wanted to do," indicating their interest. While not all responses were as detailed, most students touched on similar aspects. The most common reasons were that the projects were novel; the projects were open-ended and outcomes were uncertain; they had freedom to make their own discoveries; and the projects gave them a sense of ownership.

\section{DISCUSSION AND CONCLUSION}

Our POS item scores were inconsistent, with items 1 10 indicating students experiencing high ownership and items 11-16 indicating low ownership. However, analysis of our interviews provided evidence that students were in fact experiencing ownership, due to the presence of their interest, agency, and positive affect. But negative affect (namely frustration) was also prevalent in the interviews and was the result of students' struggles/challenges. Given that students experienced a range of emotion, it is unclear how they might have interpreted items 11-16: their responses may have been based on averaging their feelings over the project, how they felt in the moment, or if they had felt that way at all. However, what is clear, based on our interviews, is that students can have a complex affective experience, which is not captured by the POS, and yet still demonstrate ownership over their projects. In fact, this range of affect may actually be more beneficial to the development of ownership, given the right balance of negative and positive affect, than just positive experiences alone. In ongoing work, we are designing a framework that is tailored to this upper-division context and includes the dynamic nature of ownership and the complex affect that accompanies it.

\section{ACKNOWLEDGMENTS}

Special thanks to Professor Chad Hoyt at Bethel University for all his assistance with this study. This work was supported by NSF grants DUE-1323101, PHY1125844, PHY-1208930, and PHY-1560023.
[1] J. Wiley, Student Ownership of Learning : An Analysis, M.s., Universit of Hawai'i (2009).

[2] S. L. Laursen, A.-B. Hunter, E. Seymour, H. Thiry, and G. Melton, Undergraduate research in the sciences (Jossey-Bass, 2010).

[3] National Research Council, Discipline-Based Education Research: Understanding and Improving Learning in Undergraduate Science and Engineering edited by S. R. Singer, N. R. Nielsen, and H. A. Schweingruber (National Academies Press, 2012).

[4] D. I. Hanauer, J. Frederick, B. Fotinakes, and S. a. Strobel, CBE Life Sciences Education 11, 378 (2012).
[5] D. I. Hanauer and E. L. Dolan, CBE Life Sciences Education 13, 149 (2014).

[6] T. O'Neill and A. C. Barton, School Science and Mathematics 105, 292 (2005)

[7] M. Milner-Bolotin, The Effects of Topic Choice in ProjectBased Instruction on Undergraduate Physical Science Students' Interest, Ownership, and Motivation, Ph.D. thesis (2001).

[8] M. Enghag and H. Niedderer, International Journal of Science and Mathematics Education 6, 629 (2008)

[9] M. Enghag, P. Gustafsson, and G. Jonsson, International Journal of Science and Mathematics Education 7, 455 (2009) 